\section{Rnf12 and $X$ inactivation}

It has been hypothesized that there are X-linked activators of $\mathrm{X}$-chromosome inactivation ( $\mathrm{XCl}$-activators) that function in dose-dependent activation of the Xist gene, which encodes a noncoding RNA transcript that spreads over the inactive $X$ chromosome in female cells. Now, Joost Gribnau and colleagues report the first identification of an $\mathrm{X}$-linked $\mathrm{XCl}$-activator, the E3 ubiquitin ligase Rnf12 (Cell 139, 999-1011, 2009). The authors show that a transgene carrying Rnf12 triggered XCI in differentiating mouse embryonic stem (ES) cells. In male cells, transgenic expression of $R n f 12$ resulted in a cloud of Xist transcripts that accumulated on the $\mathrm{X}$ chromosome, whereas in female cells, there were two Xist clouds, indicating that both $\mathrm{X}$ chromosomes had been inactivated. Disruption of the open reading frame of Rnf12 rendered the transgene nonfunctional in inducing $\mathrm{XCl}$, confirming the identity of $\mathrm{Rnf12}$ as an $\mathrm{XCl}$-activator. Expression studies revealed that the concentration of endogenous Rnf12 is higher in female cells than in male cells, and that it is expressed at $\mathrm{XCl}$ initiation during ES cell differentiation in accordance with its proposed role. Furthermore, targeted deletion of Rnf12 in female ES cells resulted in an $80 \%$ reduction of initiation of $\mathrm{XCl}$ during differentiation; this both confirms the function of Rnf12 and indicates the likely presence of additional $\mathrm{XCl}$-activators.

\section{Maize genome and HapMap}

Patrick Schnable, Doreen Ware, Richard Wilson and colleagues report a draft sequence of the B73 maize genome (Science 326, 1112-1115, 2009). The 2.3-Gb genome was sequenced using a minimum tiling path of nearly 17,000 bacterial artificial chromosomes and 63 fosmid clones, all of which were shotgun sequenced at $4-6 \times$ coverage. 32,540 protein-encoding and 150 miRNA genes were predicted in 11,892 gene families. The large size of the maize genome is attributable to the proliferation of long terminal repeat retrotransposons; almost $85 \%$ of the $\mathrm{B} 73$ reference genome is composed of maize transposable elements, which are distributed in a nonuniform manner throughout the genome. Michael Gore, Jer-Meng Chia, Edward Buckler and colleagues report the first-generation haplotype map of maize (Science 326, 1115-1117, 2009). The authors generated more than 1 billion short-sequencing reads from a diverse panel of 27 inbred lines that are representative of the worldwide diversity in maize. The authors identified 3.3 million polymorphisms and find that haplotypes are highly divergent and recombination rates are variable across the genome. Because linkage disequilibrium in maize decays within 2,000 bp, the authors conclude that GWAS studies in maize will require an increase in the number of available markers by an order of magnitude.

$P C$

\section{DICER1 haploinsufficiency in cancer}

Recent studies in mice have shown that global defects in miRNA processing can promote tumorigenesis. Tyler Jacks and colleagues (Genes Dev. 23, 2700-2704, 2009) now report several additional lines of evidence indicating that the miRNA-processing enzyme Dicer1 functions as a haploinsufficient tumor suppressor. Building on earlier work (Nat. Genet. 39, 673-677, 2007), the authors examined the effects of Dicer1 deletion in two different mouse cancer models and found that mice carrying one conditional Dicer 1 allele were more tumor prone than mice carrying two conditional Dicer1 alleles. In both models, the tumors that developed in mice carrying one conditional Dicer 1 allele showed retention of the wild-type allele. Similarly, the tumors that developed in mice carrying two copies of the conditional Dicerl allele were found to have undergone incomplete recombination, suggesting the presence of selection against complete loss of Dicer1 in these tumors. The authors also surveyed copy number changes across several human cancers and found that hemizygous loss of DICER 1 was frequent in many cancers, including breast, colorectal, lung and kidney. Taken together, these findings suggest that DICER1 haploinsufficiency may be an important driver in human cancer. $K V$

\section{Mixed-lineage leukemia and transcriptional elongation}

Mixed-lineage leukemia is an aggressive type of leukemia that is often caused by chromosomal translocations that lead to fusion proteins involving the DNA binding protein histone $\mathrm{H} 3$ lysine 4-specific methyltransferase (MLL). MLL fusions are known to be oncogenic transcription factors that upregulate expression of target genes that ultimately block hematopoietic differentiation and transform hematopoietic cells, but the molecular mechanisms underlying the transforming properties of MLL fusions are not well understood. Robert Slany and colleagues now report that MLL fusion proteins target the transcriptional elongation complex or elongation assisting proteins (EAP) (PLoS Biol. 7, e1000249, 2009). MLL fusions become integrated into the EAP complex, leading to constitutive recruitment of EAP components at MLL-fusion targets and stimulation of transcriptional elongation of those targets. Using time-resolved chromatin immunoprecipitation, the authors found that MLL fusion proteins led to the sustained presence of elongating RNA polymerase II and to decreased methylation of the inhibitory chromatin marks H3K9 and H3K27 on target chromatin. The authors find that MLL fusion-transformed cells show sensitivity to pharmacological inhibition of EAP activity and suggest that drugs that target transcriptional elongation should be explored as possible treatments for mixed-lineage leukemia.

PC

\section{Salmonella epidemic}

Salmonella enterica serovar Typhimurium, one of the most common serovars associated with human and animal disease, has been associated with the epidemic of highly invasive nontyphoidal salmonella disease in sub-Saharan Africa. Robert Kingsley and colleagues now report comparative genomic analyses of $S$. typhimurium strains sampled from within this epidemic, including 31 Malawian and 20 Kenyan isolates (Genome Res. published online 9 November, 2009; doi: 10.1101/ gr.091017.109). Multilocus sequence typing of these strains and comparisons to global isolates showed that most of the new isolates defined a distinct sequence type, ST313, that is rare outside Africa. They report the whole genome sequence of a representative ST313 strain isolated from Blantyre, Malawi in 2004 during the peak of the epidemic. This report showed evidence of genome degradation, including loss of genes implicated in host-pathogen interactions. They also report draft whole-genome sequences of two ST313 isolates: a drug-sensitive strain isolated from Blantyre in 1997 before the emergence of resistance and a multi-drug-resistant (MDR) strain isolated from Nairobi, Kenya in 2003 during the MDR epidemic. Examination of the Blantyre strains suggested that the emergence of MDR during this epidemic was caused by clonal replacement. 